\title{
Deconstructing Amazon EC2 Spot Instance Pricing
}

\author{
Orna Agmon Ben-Yehuda \\ Muli Ben-Yehuda \\ Assaf Schuster \\ Dan Tsafrir \\ Computer Science Department \\ Technion - Israel Institute of Technology \\ Haifa, Israel \\ \{ladypine, muli, assaf, dan\}@cs.technion.ac.il
}

\begin{abstract}
Cloud providers possessing large quantities of spare capacity must either incentivize clients to purchase it or suffer losses. Amazon is the first cloud provider to address this challenge, by allowing clients to bid on spare capacity and by granting resources to bidders while their bids exceed a periodically changing spot price. Amazon publicizes the spot price but does not disclose how it is determined.

By analyzing the spot price histories of Amazon's EC2 cloud, we reverse engineer how prices are set and construct a model that generates prices consistent with existing price traces. We find that prices are usually not market-driven as sometimes previously assumed. Rather, they are typically generated at random from within a tight price interval via a dynamic hidden reserve price. Our model could help clients make informed bids, cloud providers design profitable systems, and researchers design pricing algorithms.
\end{abstract}

Keywords-cloud; spot price; spot instance; Amazon EC2

\section{INTRODUCTION}

Unsold cloud capacity is wasted capacity, so cloud providers would naturally like to sell it. Clients might be enticed to purchase it if they are provided with enough incentive, notably, a cheaper price. In late 2009, Amazon was the first cloud provider to attempt to provide such an incentive by announcing its spot instances pricing system. "Spot Instances [...] allow customers to bid on unused Amazon EC2 capacity and run those instances for as long as their bid exceeds the current Spot Price. The Spot Price changes periodically based on supply and demand, and customers whose bids exceeds it gain access to the available Spot Instances" [1]. With this system, Amazon motivates purchasing cheaper capacity while ensuring it can continuously act in its best interest by maintaining control over the spot price. Section II summarizes the publicly available information regarding Amazon's pricing system.

Amazon does not disclose its underlying pricing policies. Despite much interest from outside Amazon [2]-[4], its spot pricing scheme has not, until now, been deciphered. The only information that Amazon does reveal is its temporal spot prices, which must be publicized to make the pricing system work. While Amazon provides only its most recent price history, interested parties record and accumulate all the data ever published by Amazon, making it available on the Web [5], [6]. We leverage the resulting trace files for this study. The trace files, along with the methodology we employ to use them, are described in Section III.

Knowing how a leading cloud provider like Amazon prices its unused capacity is of potential interest to both cloud providers and cloud clients. Understanding the considerations, policies, and mechanisms involved may allow other providers to better compete and to utilize their own unused capacity more effectively. Clients can likewise exploit this knowledge to optimize their bids, to predict how long their spot instances would be able to run, and to reason about when to purchase cheaper or costlier capacity.

Motivated by these benefits, we attempt in Sections IV$\mathbf{V}$ to uncover how Amazon prices its unused EC2 capacity. We construct a spare capacity pricing model and present evidence suggesting that prices are typically not determined according to Amazon's public definition of the spot pricing system as quoted above. Rather, the evidence suggests that spot prices are usually drawn from a tight, fixed price interval, reflecting a random reserve price that is not driven by supply and demand. (A reserve price is a hidden price below which bids are ignored.) Consequently, published spot prices reveal little about actual, real-life client bids; studies that assume otherwise (e.g., [7], [8]) are, in our view, misguided and their results questionable. We speculate that Amazon utilizes such a price interval because its spare capacity usually exceeds the demand.

In Section VI we put our model to the test by conducting pricing simulations and by showing their results are consistent with EC2 price traces. We then discuss the possible benefits of using dynamic reserve price systems (such as the one we believe is used by Amazon) in Section VII. Finally, we survey the related work in Section VIII and offer some concluding remarks in Section IX.

\section{Pricing Cloud Instances}

Amazon's EC2 clients rent virtual machines called instances, such that each instance has a type describing its computational resources as follows: m1.small, m1.large and $\mathrm{m} 1 . x$ large, respectively denote small, large, and extra large "standard" instances; m2.xlarge, m2.2xlarge, and m2.4xlarge respectively denote extra large, double extra large, and quadruple extra large "high memory" instances; 
and c1.medium and c1.xlarge respectively denote medium and extra-large "high CPU" instances.

An instance is rented within a geographical region. We use data from four EC2 regions: us-east, us-west, eu-west and ap-southeast, which correspond to Amazon's data centers in Virginia, California, Ireland, and Singapore.

Amazon offers three purchasing models, all requiring a fee from a few cents to a few dollars, per hour, per running instance. The models provide different assurances regarding when instances can be launched and terminated. Paying a yearly fee (of hundreds to thousands of dollars) buys clients the ability to launch one reserved instance whenever they wish. Clients may instead choose to forgo the yearly fee and attempt to purchase an on-demand instance when they need it, at a higher hourly fee and with no guarantee that launching will be possible at any given time. Both reserved and on-demand instances remain active until terminated by the client.

The third, cheapest purchasing model provides no guarantee regarding either launch or termination time. When placing a request for a spot instance, clients bid the maximum hourly price they are willing to pay for running it (called declared price or bid). The request is granted if the bid is higher than the spot price; otherwise it waits. Periodically, Amazon publishes a new spot price and launches all waiting instance requests with a maximum price exceeding this value; the instances will run until clients terminate them or the spot price increases above their maximum price. All running spot instances incur a uniform hourly charge, which is the current spot price. The charge is in full hours, unless the instance was terminated due to a spot price change, in which case the last fraction of an hour is free of charge.

In this work, we assume that instances with bids equal to the spot price are treated similarly to instances with bids higher than the spot price.

\section{Methodology}

Trace Files: We analyze $64(=8 \times 4 \times 2)$ spot price trace files associated with the 8 aforementioned instance types, the 4 aforementioned regions, and 2 operating systems (Linux and Windows). The traces were collected by Lossen [5] and Vermeersch [6]. They start as early as November 30, 2009 (traces for region ap-southeast are only available from the end of April 2010). In this paper, unless otherwise stated, we use data accumulated until July 13, 2010,

Availability: We define the availability of a declared price as the fraction of the time in which the spot price was equal to or lower than that declared price. Formally, a persistent request is a series of requests for an instance that is immediately re-requested every time it is terminated due to the spot price rising above its bid. Given a declared price $D$, we define $D$ 's availability to be the time fraction in which a persistently requested instance would run if $D$ is its declared price. Formally, let $H$ be a spot price trace file, and let $T_{b}$ and $T_{e}$ be the beginning and end of a time interval within $H$. The availability of $D$ within $H$ during $\left[T_{b}, T_{e}\right]$ is:

$$
\text { availability }\left.^{H}(D)\right|_{\left[T_{b}, T_{e}\right]}=\frac{T_{b \rightarrow e}^{H}(D)}{T_{e}-T_{b}}
$$

where $T_{b \rightarrow e}^{H}(D)$ denotes the time between $T_{b}$ and $T_{e}$ during which the spot price was lower than or equal to $D$. The availability of price $D$ reflects the probability that spot instances with this bid would be immediately launched when requested at some uniformly random time within $\left[T_{b}, T_{e}\right]$.

\section{EVIDENCE FOR ARTIFICIAL PRICING INTERVENTION}

\section{A. Market-Driven Auctions}

Amazon's description of "How Spot Instances Work" [1] gives the impression that spot prices are set through a uniform price, sealed-bid, market-driven auction. "Uniform price" means all bidders pay the same price. "Sealed-bid" means bids are unknown to other bidders. "Market-driven" means the spot price is set according to the clients' bids. One example of such an auction is an $(N+1)^{t h}$ price auction of multiple goods, with retroactive supply limitation (after clients bid), to maximize the provider's revenue. However, Amazon might be using some other mechanism consistent with their description.

In an $(N+1)^{t h}$ price auction of multiple goods, each client bids for a single good (i.e., a spot instance). The provider chooses the top $N$ bidders. The provider may set $N$ up-front on the basis of available capacity. The provider might retroactively set $N$ after receiving the bids, to maximize revenue. In any case, $N$ cannot exceed the available capacity. The provider sets the uniform price to the price declared by the highest bidder who did not win the auction (bidder number $N+1$ ) and publishes it. The top $N$ winning bidders pay the published price and their instances start running. In this case, the published price is a price bid by an actual client.

The provider may also decide to ignore bids below a publicly known minimal price or below a hidden reserve price (no relation to reserved instances), to prevent the goods from being sold cheaply, or to give the impression of increased demand.

We conjecture that usually, contrary to impressions conveyed by Amazon [1] and assumptions made by researchers [7], [8], the spot price is set according to a constantly changing reserve price, disregarding client bids. In other words, most of the time the spot price is not marketdriven but is set by Amazon according to an undisclosed algorithm.

\section{B. Evidence: Availability as a Function of Price}

In support of this conjecture, we analyze the relationship between an instance's declared price (how much a client would be willing to pay for it) and the resulting availability 
between January 20th and July 13th, 2010. Fig. 1 shows the availability of different spot instance types as a function of declared price (price-availability graphs), for all examined Windows spot instance types in all regions. Results for instances running Linux (not shown) are qualitatively similar. The prices of different resources seem unrelated, except that they share the same functional shape: a sharp linear increase in availability until a knee (sharp change in slope) is reached. The knee is usually high, representing an availability of 0.95 or more. After the knee, the availability grows with declared price but at a slower, non-linear rate.

Fig. 2 shows normalized price-availability graphs for Linux: prices on the horizontal axis are normalized by their instance-type's respective on-demand prices. We see that Linux types can be classified by region. Each of the two region classes has a distinct normalized price range in which the availability's dependency on the price is linear. One class contains us-east, and the other class contains the other regions.

Fig. 3 shows the data presented in Fig. 1 as normalized price-availability graphs. As in Fig. 2, different types can be classified by region: us-east or all other regions. Not as in Fig. 2, different types have different normalized prices within a class, and the relative price difference between any type pair is the same in each class. The m1.small type, indicated in Fig. 3 by an arrow, has a particularly low knee, with an availability of 0.45 . Figs.. 1-3 show that availability strongly depends on declared price for all regions and all instance types, and that this dependency has a typical recurring shape, which can be explained by assuming that Amazon uses the same mechanism to set the price in different regions. The particular shape of the dependency could be explained in one of two ways: either Amazon's spot prices reflect real client bids and the shaped dependency occurs naturally, or the spot prices are the result of a dynamic hidden reserve price algorithm, of which the shaped dependency is an artifact.

Let us first consider the assumption that the shaped dependency occurs naturally due to real client bids. The differences between absolute price ranges of the same type in different regions (Fig. 1) show that different regions experience different supply and demand conditions. This means that uncoordinated client bids for different types and regions would have to naturally and independently create all of the following phenomena: (1) normalized prices turning out identical for various Linux types but different for Windows types; (2) a rigid linear connection between availability and price that turns out to be identical for different types and regions; (3) a distinct region having a normalized price range different than all the rest (which turn out to have identical ranges); and (4) normalized prices for Windows instances which differ from one another by identical amounts in each of the two region classes, creating the same pattern for both.

For the sake of argument, let us also consider the possibil- ity that a conspiring group of clients have already reverseengineered Amazon's algorithm and submit coordinated bids that cause the aforementioned phenomena. Since the phenomena we describe can be seen in all 64 analyzed traces, these clients would have to consume a sizable share of the spot instance supply in all 64 resources, bidding low bids (which would then eventually become the spot price). This would systematically limit the supply available to the many different legitimate clients known to use EC2 spot instances. When these clients then bid higher than the spot price, the spot price rises, terminating the conspiring clients' instances. From this point on, the conspiring clients' effect on the spot price is limited. Furthermore, customers must have Amazon's approval for the purchase of spot instances beyond the first 100 . Hence, we consider this explanation highly unlikely.

Our hypothesis: we consider it unlikely that all four phenomena could have resulted from Amazon setting the price solely on the basis of client bids. We therefore lean towards the hypothesis that Amazon uses a dynamic algorithm, independent of client bids, to set a reserve price for the auction, that the auction's result is usually identical to the reserve price, and that the prices Amazon announces are therefore usually not market-driven. Both the simulation results presented in Section VI and Occam's razor-preferring the simplest explanation-support this hypothesis.

If our hypothesis is correct, then all four phenomena listed above are easily explained by a dynamic reserve price algorithm which gets as input prices normalized by respective on-demand prices. This input is different for the us-east region, for different sets of types, and for different operating systems.

\section{Dynamic Random Reserve Price}

First we will characterize the requirements for a dynamic reserve price algorithm that will be consistent with the published EC2 price traces. Then we will construct such an algorithm, and propose it as a candidate for the algorithm behind the EC2 pricing.

We contend that for each spot instance type, the dynamic reserve price algorithm gets as input a floor price $F$ and a ceiling price $C$, expressed as fractions of the on-demand price. The floor price is the minimal price, exemplified in Fig. 1. The ceiling price is the price corresponding to the knee in the graph (shown in the same figure), or the maximal price if no knee exists. The algorithm dynamically changes the reserve price such that there is a linear relation between availability and prices in the pricing band (the floor-ceiling range). It guarantees that the reserve price is kept within the band.

We deconstruct the reserve price algorithm using traces from April-July, 2010, when the spot price in eight apsoutheast.windows instance types almost always stayed within the pricing band. We matched the price changes in 


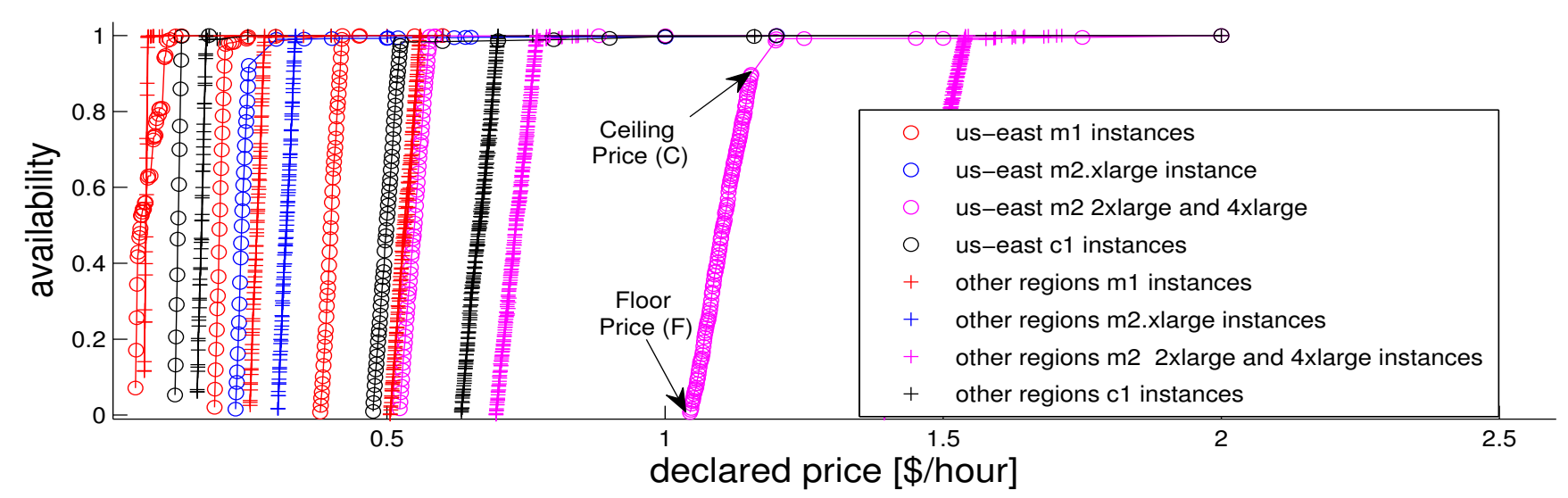

Figure 1: Availability of Windows-running spot instance types as a function of their declared price. The legend is multiplexed: us-west, eu-west, ap-southeast all appear in the legend as "other regions". m1.small, m1.large and m1.xlarge all appear as $\mathrm{m} 1$. c1.medium and c1.xlarge appear as $\mathrm{c} 1$.

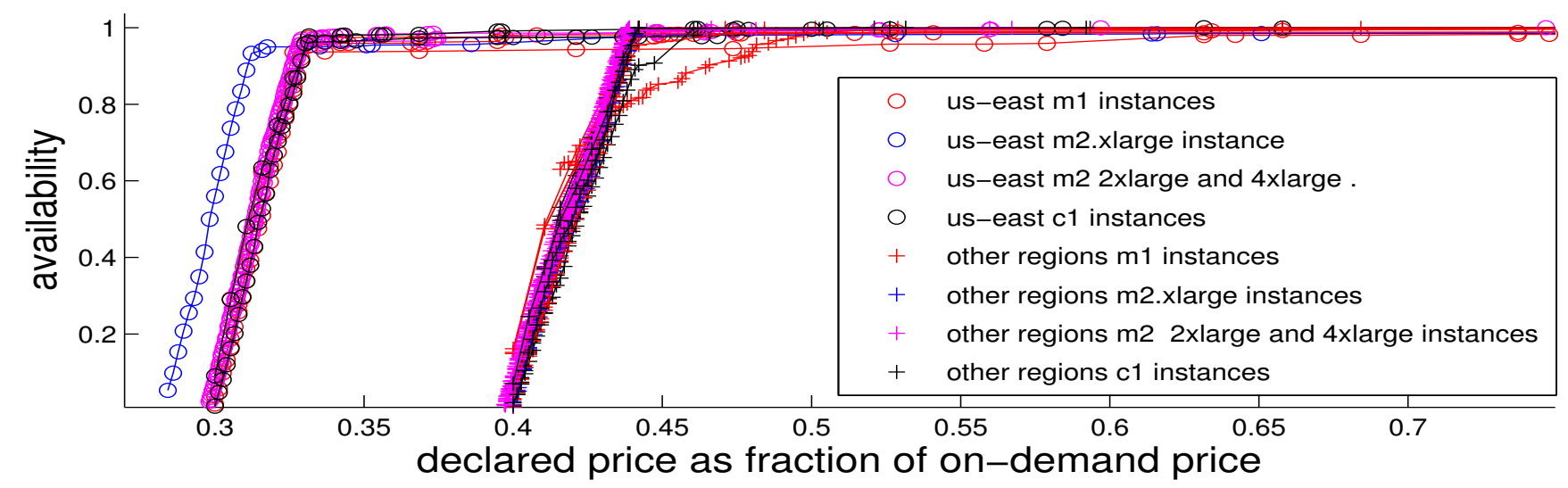

Figure 2: Availability of Linux-running spot instance types as a function of their normalized declared price. The declared price is divided by the price of a similar on-demand instance. The legend is multiplexed as in Fig. 1. Most of the curves overlap.

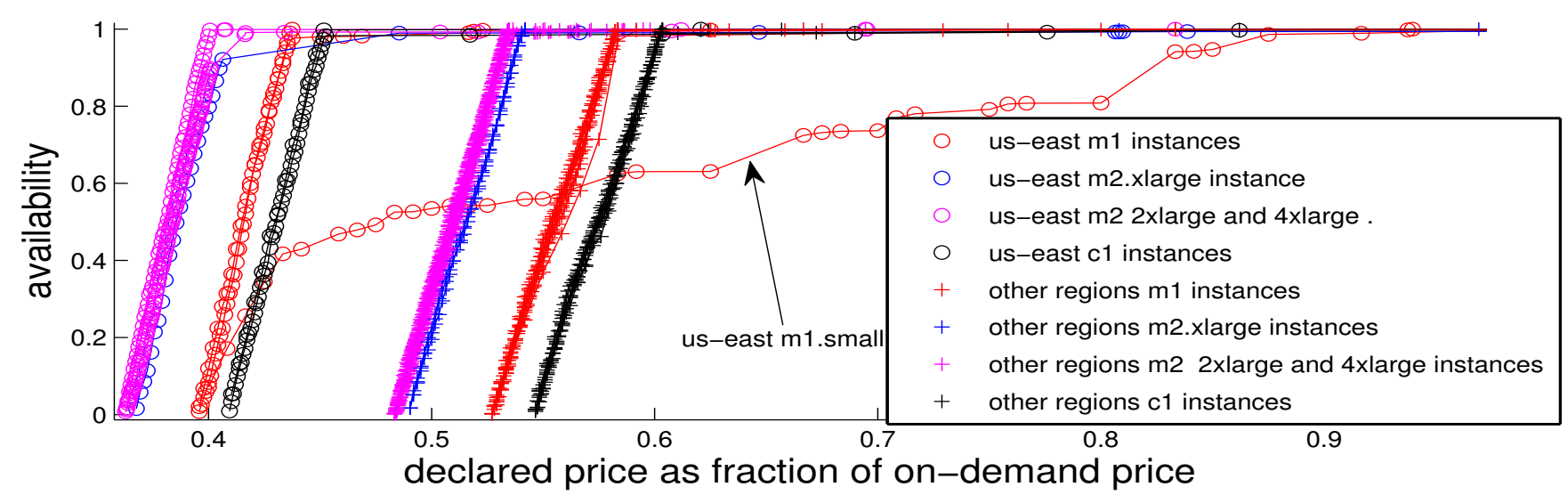

Figure 3: Availability of Windows-running spot instance types as a function of their normalized declared price. The declared price is divided by the price of a similar on-demand instance. The legend is multiplexed as in Fig. 1. Many of the curves overlap. us-east.windows.m1.small is indicated by an arrow. 


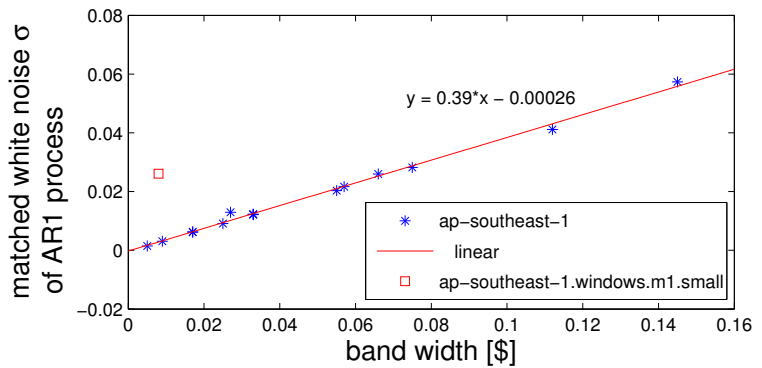

Figure 4: Standard deviation of matched $A R(1)$ process as a function of pricing band width.

those traces (denoted by $\Delta$ ) with an $A R(1)$ (auto-regressive) process .We found a good match (i.e., negligible coefficients of higher orders $a_{i}$ for $i>1$ ) to the following process:

$$
\Delta_{i}=-a_{1} \Delta_{i-1}+\epsilon(\sigma),
$$

where $a_{1}=0.7$ and $\epsilon(\sigma)$ is white noise with a standard deviation $\sigma$. We matched $\sigma$ with a value of $0.39(C-F)$. These parameters fit all the analyzed types except $\mathrm{m} 1$.small, which matched different values $\left(a_{1}=0.5, \sigma=0.5(C-\right.$ $F)$ ). The standard deviations are given in Fig. 4. This close fit-the same parameters characterizing the randomness of several different traces-is consistent with our hypothesis that the prices are usually set by an artificial algorithm.

Prices within the band might also result from clients bidding within the band (although others have already noted that such bids are not cost-effective [2], [4]), but mean price analysis indicates otherwise. Since an $A R(1)$ process is symmetric, its theoretic average is the middle of the band. For the 8 traces we used here, the average price was $98 \%$ $100 \%$ of the middle of the band. This, too, supports our hypothesis that the spot price within the band is almost always determined solely by the $A R(1)$ process, i.e., is equal to the reserve price. In addition, we find that on average over all the 64 traces we analyzed, prices are within the band $98 \%$ of the time. We conclude that prices are determined artificially by an $A R(1)$ reserve price algorithm and do not represent real client bids around $98 \%$ of the time.

On the basis of this analysis, we construct the $A R(1)$ reserve price algorithm: The first two reserve prices are defined as $P_{-1}=F+0.1(C-F), P_{0}=F$. The following prices are defined as $P_{i}=P_{i-1}+\Delta_{i}$, where $\Delta_{i}=-0.7 \cdot \Delta_{i-1}+\epsilon(0.39 \cdot(C-F))$. The process is truncated to the $[F, C]$ range by regenerating the white noise component while $P_{i}$ is outside the $[F, C]$ range or identical to $P_{i-1}$. All prices are rounded to one-tenth of a cent, as done by Amazon during 2010.

Fig. 5 provides a spectral analysis of one of the Amazon traces and of prices produced by our $A R(1)$ algorithm. The match shows that our reverse-engineered reserve price algorithm is consistent with Amazon's algorithm.

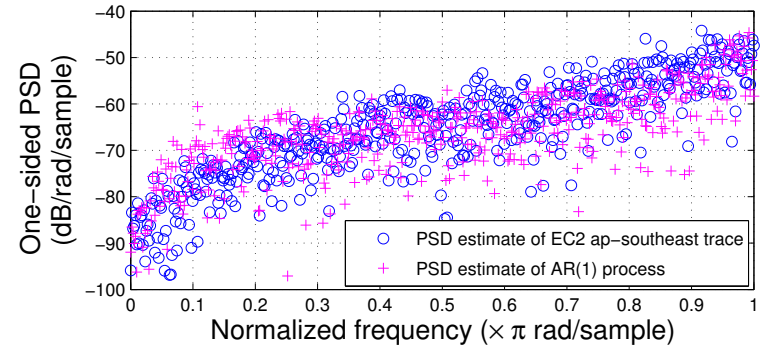

Figure 5: Power spectral density (Periodogram) estimate of an EC2 price trace, and our derived $A R(1)$ price trace.

\section{PRICING EPOCHS}

To statistically analyze spot price histories, it would be erroneous to assume that the same pricing model applies to all the data in the history trace. Rather, each trace is divided to contiguous epochs associated with different pricing policies. We show here that our main traces are divided into three parts as depicted in Fig. 6. Since the pricing mechanism changes notably and qualitatively between epochs, data regarding these epochs should be separated if an associated statistical analysis is to be sound. Accordingly, for the purpose of evaluating the effectiveness of client algorithms, strategies, and predictions, the data from a (single) epoch of interest should be used.

The first epoch starts, according to our analysis, as early as November 30th, 2009 and ends on December 14th, 2009, the date on which Amazon announced the availability of spot instances. During this time instances were unknown to the general public. Hence, the population which undertook any bidding during the first epoch was smaller than the general public, of nearly constant size, and possibly had additional information regarding the internals of the pricing mechanism at that time.

The second epoch begins with the public announcement on December 14th, 2009. It ends with a pricing mechanism change around January 8th, 2010, when minimal spot prices suddenly change in most traces (usually decrease, though Fig. 6 demonstrates an increase). It is characterized by long intervals of constant low prices.

The third epoch begins on January 20th, 2010. Instance types and regions began to change minimal price around January 8th, but we define the beginning of the epoch as the date in which the last one (eu-west.linux.m2.2xlarge) reached a new minimal price. Due to (1) the gradual move to the new minimal values and to (2) a bug in the pricing mechanism that was fixed in mid-January 2010 [9], we choose to disregard data from the transition period between the second and third epochs.

Additional epoch-defining dates are dates when the pricechange timing algorithm was changed, e.g., July 25th, 2010 and February 9th, 2011 for the us-east region (see Section VI). 


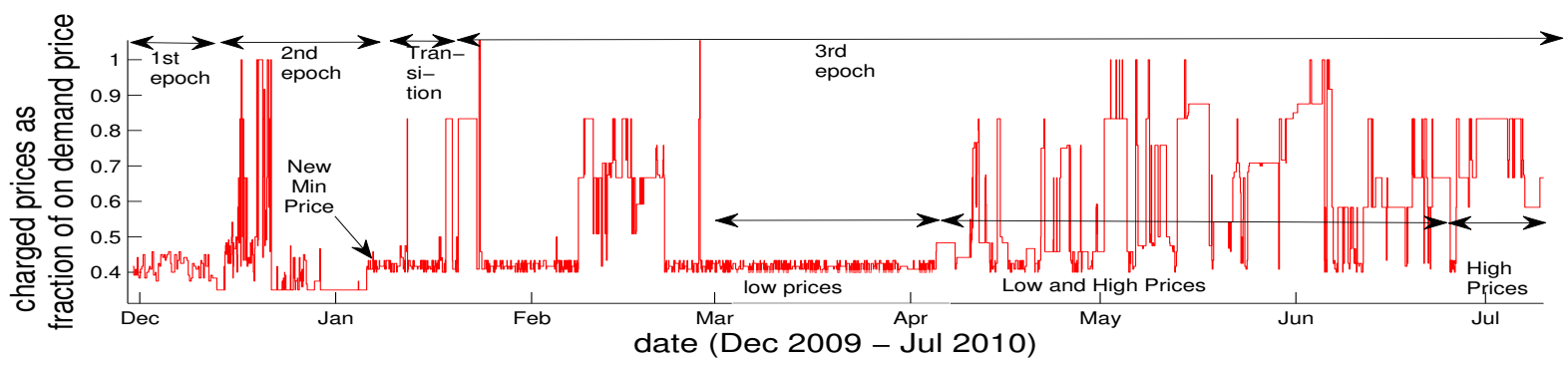

Figure 6: Price history for us-east.windows.m1.small. Three time epochs are shown, with a transition period between the second and third epochs. The spot price is presented as a fraction of the on-demand price for the same instance.

These abrupt time-correlated changes in many regions and instance types further support our hypothesis, since prices are likely to undergo coordinated and abrupt changes precisely where they are artificial.

\section{Spot Price Simulation}

To get a better feel for the validity of our hypothesis, we simulated the prices and availability resulting from setting the price via a sealed-bid $(N+1)^{t h}$ price auction with a reserve price with retroactive supply limitation, as described in Section IV-A. The on-demand price was defined as 1 . The reserve price was either constant (0.4) or the $A R(1)$ algorithm defined in Section IV-C, with a band of [0.4, 0.45].

Workload Modeling: In the absence of cloud workload traces, we fed the simulation with $20 \mathrm{~K}$ tasks, with runtimes in the range of 10 minutes to 24 hours, out of the LPC-EGEE cluster workload from 2004, kindly provided by Medernach [10] via the Parallel Workloads Archive [11]. LPC-EGEE is characterized by tasks which are small in comparison to the capacity of the cluster, allowing for elasticity. In the simulation, each task was interpreted as a single instance, submitted at the same time and requiring the same amount of run-time as in the original trace to complete.

Customer Bid Modeling: Due to the lack of information on the distribution of real client bids (since we argue that Amazon's price traces supply little information of this type), we compare several bidding models, and verify that the qualitative results are insensitive to the bid modeling. All the distributions were adjusted to uniform minimal and ondemand prices.

The first model is a Pareto distribution (a widely applicable economic distribution [12], [13]) with a minimal value of 0.4 , and a Pareto index of 2 , a reasonable value for income distribution [12]. The second model is $\mathcal{N}\left(0.7,0.3^{2}\right)$, truncated at 0.4. The third is a linear mapping from runtimes to $(0.4,1]$, which reflects client aversion to having longrunning instances terminated.

Price Change Timing: Price changes in the simulation are triggered according to the CDF of intervals between them, collected during January-July 2010, and given in Fig. 7 (solid line). This period was characterized by quiet times-prices never changed before 60 minutes or between

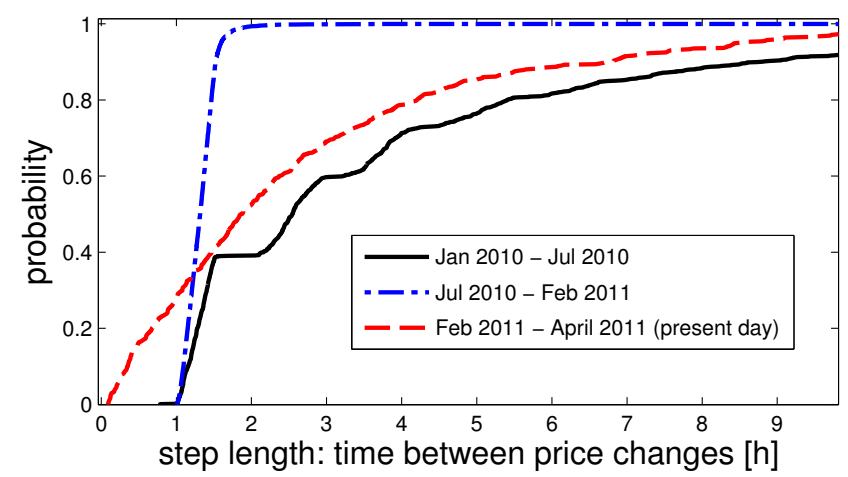

Figure 7: CDF of time interval between price changes for different versions of the price change scheduling algorithm. Input: us-east.linux.m1.small

90 and 120 minutes since the previous price change. It is interesting to note that such quiet times can be monetized by clients to gain free computation power with a probability of about $25 \%$, by submitting an instance with a bid of the current spot price 31 minutes after a price change. The instance would then have a $50 \%$ possibility of undergoing another price change within 30-60 minutes. If that change is a price increase, the instance would be terminated, and the client would gain, on average, 45 minutes of free computation. Clients do not exploit this loophole in our simulation.

Fig. 7 also presents the evolution of the timing of price changes for the us-east region. The next algorithm (in place from July, 2010 until Feb 8th, 2011) allowed for a quiet hour after a price change. The following one (starting Feb 9th, 2011) matches an exponential distribution with a 1.5 hour rate parameter, with five quiet minutes. This almost memoryless algorithm prevents abuse of the timing algorithm.

Simulator Event-Driven Loop: We created a tracebased event-driven simulator, where events are: (1) instance submission and termination and (2) price changes (due to a scheduled change or to a waiting instance with a bid higher than the spot price). We ran the simulation on 70 CPUs, according to the number in the LPC-EGEE trace. We ended the simulation when the last job had been submitted.

Simulation Results: Simulation results in terms of price-availability graphs are presented in Fig. 8, for different 


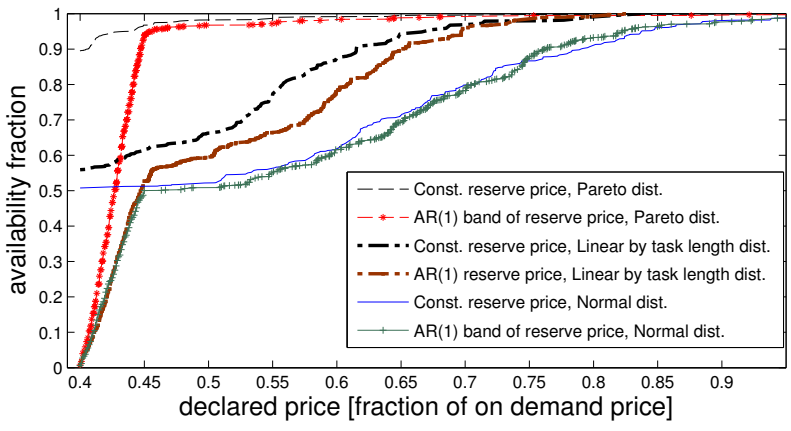

Figure 8: Simulation results for various bidding models, with constant and $A R(1)$ reserve price.

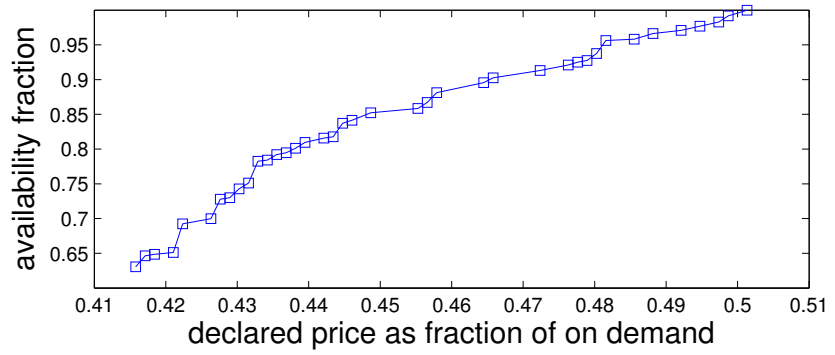

Figure 9: Availability as a function of the declared price during the second epoch for us-west.linux.m1.xlarge.

bid models and price setting mechanisms. The functions of simulations with the $A R(1)$ reserve price feature a linear segment and a knee in high availability, as do the availability functions of EC2 during the third epoch, which are shown in Figs. 1, 2, and 3. The constant reserve price functions do not exhibit this behavior. Rather, they are jittery, like the high price regime of the us-east.windows.m1.small graph in Fig. 3, and the second epoch graph in Fig. 9.

Furthermore, the availability of the reserve price in the constant reserve price simulations is high $(0.5,0.56,0.9)$, as it is in the second epoch (0.63 in Fig. 9). In contrast, the availability of the minimal price in the $A R(1)$ reserve price simulations and in the third epoch tends to zero as the number of discrete prices within the band grows.

We consider these simulation results another indication that most prices in the EC2 traces during the third epoch are set using an artificial, non-market-driven algorithm, in particular an $\mathrm{AR}(1)$ reserve price. The simulation results also suggest that Amazon set prices via a market-driven auction with a constant reserve price during the second epoch (December, 2009 until January, 2010), and that prices above the band are market-driven.

\section{Dynamic Reserve PRICE Benefits}

The dynamic $A R(1)$ reserve price mechanism has several long-term, wide-range, benefits that may justify its use.

Like a constant minimal or reserve price, it guarantees that on-demand instances are not completely cannibalized by spot instances. Yet it also allows the provider to sell instances on machines which would otherwise run idle, to provide elasticity for the fixed price instances. Spot instances, which can be quickly evacuated, still reduce the costs associated with idle servers, with no real harm to the main offering of on-demand instances.

Katkar and Reiley [14] found that for low-priced eBay sales of up to $\$ 20$, (hidden) reserve prices deter good clients and yield lower revenues than minimal (published) prices. However, the advantage of the (hidden) reserve price is that the provider can set it using a random algorithm, with no obligation to inform clients. A dynamic reserve price is better than a constant minimal price, because it maintains an impression of constant change, thus preventing clients from becoming complacent. It forces them to either bid higher than the band or tolerate sudden unavailability. It also serves to occasionally clear queues of low bids within the band, a purpose that is not served by a constant reserve price that is equal to the ceiling price.

A random reserve price might also serve other goals, if the public is unaware of its use. By creating an impression of false activity (demand and supply changes), the random reserve price can mask times of low demand and price inactivity, thus possibly driving up the provider's stock. A large enough band covering the spectrum of probable prices could also mask high demand and low supply, and thus help to maintain the illusion of an infinitely elastic cloud. However, if the pricing band is relatively small, as in the case of Amazon EC2 spot prices, the provider's use of an $A R(1)$ process for setting the price within the band is a strong indication of low demand.

\section{RELATED WORK}

Optimizing Client Goals Using Spot Price Traces: Andrzejak, Kondo and Yi used spot price histories to advise the client how to minimize monetary costs while meeting an SLA [15], and to schedule checkpoints [16] and migrations [17]. The first two works used data from the transition period between the second and third epoch, and focused on eu-west, which suffered most from the transition. The last interchangeably used data from before and after the change in the price change algorithm on July 25, 2010.

Mattess, Vecchiola and Buyya [4] examined client strategies for using spot instances to manage peak loads. They evaluated the strategies using an EC2 spot instance trace of the third epoch only, attributing the different trace behavior prior to January 18th, 2010 to Christmas and to the recent introduction of spot instances. They identified the price band and recommended bidding right under the on-demand price, noting that bidding just above the band is almost as good.

Chohan et al. [2] note the cost-effectiveness of bidding at the top of the band. They analyze price histories from the third epoch only, because of a pricing bug, which was fixed in mid-January 2010 [9]. The bug allowed a region to have a low price while in at least one availability zone in 
that region instances with higher prices were terminated due to congestion. The authors attributed the qualitative change of prices between the second and third epoch to the bug fix. However, this bug fix is unlikely to have caused the qualitative price changes we observe during January 2010.

Optimizing Provider Algorithms Using Spot Traces:

Zhang et al. [7] assumed Amazon uses a market-driven auction, and hence concluded that spot prices reflect actual client bids. On this basis, they sought resource allocations which optimized the provider's revenue. Chen et al. [8] assumed EC2 price traces represent market clearing prices. We doubt these assumptions in light of our findings that $98 \%$ of the time, on average, EC2 price traces are the reserve prices, and as such do not provide a lot of information about real client bids, nor are necessarily clearing prices.

Free Spot and Futures Markets: While Amazon is currently the only provider offering "spot instances," free computing resource markets have already been analyzed [18][20]. Price traces of such free markets [18], [19] differ from EC2 spot price traces: they do not have a hard minimal price and are not anchored in the bottom of the price range. Rahman, Lu and Gupta [21] evaluate free spot market options using EC2 traces, and note that the "data does not show enough fluctuations as expected in a free market."

\section{CONClusions}

Amazon EC2 spot price traces provide more information about Amazon than about its clients. We have shown that it is likely that Amazon sets spot prices using a random $A R(1)$ (hidden) reserve price which might be the basis of a marketdriven mechanism, such that high prices may reflect market changes, but most low prices, within a band of prices, are usually indicative only of the dynamic reserve price.

Understanding how Amazon prices its spare capacity is useful for clients, who can decide how much to bid for instances; for providers, who can learn how to build more profitable systems; and for researchers, who can differentiate between prices set by an artificial process and prices likely to have been set by real client bids. We have shown that many price trace characteristics (e.g., minimal value, band width, change timing) are artificial, and might change according to Amazon's decisions. Thus, researchers should be aware of the epochs present in their traces when using those traces to model future price behavior or to evaluate client algorithm performance. We have shown that indiscriminately using Amazon's current traces to model client behavior is unfounded on average $98 \%$ of the time.

\section{REFERENCES}

[1] "Amazon EC2 spot instances," http://aws.amazon.com/ec2/ spot-instances/, [accessed Aug, 2011].

[2] N. Chohan, C. Castillo, M. Spreitzer, M. Steinder, A. Tantawi, and C. Krintz, "See spot run: using spot instances for mapreduce workflows," in USENIX Conference on Hot Topics in Cloud Computing (HotCloud), 2010.
[3] D. Samovskiy, "Unexpected similarities in EC2 spot price history between regions," http://tinyurl.com/somic, Dec 2010, [accessed Apr, 2011].

[4] M. Mattess, C. Vecchiola, and R. Buyya, "Managing peak loads by leasing cloud infrastructure services from a spot market," in IEEE Int'l Conference on High Performance Computing and Communications (HPCC), 2010.

[5] T. Lossen, "Cloud exchange," http://cloudexchange.org/, [accessed Apr, 2011].

[6] K. Vermeersch, "Spot watch," http://spotwatch.eu/input/, accessed Apr, 2011.

[7] Q. Zhang, E. Gurses, R. Boutaba, and J. Xiao, "Dynamic resource allocation for spot markets in clouds," in Workshop on Hot Topics in Management of Internet, Cloud, and Enterprise Networks and Services (Hot-ICE), 2011.

[8] J. Chen, C. Wang, B. B. Zhou, L. Sun, Y. C. Lee, and A. Y. Zomaya, "Tradeoffs between profit and customer satisfaction for service provisioning in the cloud," in HPDC, 2011.

[9] "Spot instance termination conditions?" http://tinyurl.com/ 2dzp734, Mar 2010, online AWS Developer Forums discussion, accessed Apr, 2011.

[10] E. Medernach, "Workload analysis of a cluster in a grid environment," in Workshop on Job Scheduling Strategies for Parallel Processing, 2005.

[11] D. Feitelson, "Parallel workloads archive," Website, http:// www.cs.huji.ac.il/labs/parallel/workload/index.html.

[12] W. Souma, "Physics of personal income," 2002. [Online]. Available: http://arxiv.org/pdf/cond-mat/0202388

[13] M. Levy and S. Solomon, "New evidence for the power-law distribution of wealth," Physica A, vol. 242, pp. 90-94, 1997.

[14] R. Katkar and D. H. Reiley, "Public versus secret reserve prices in ebay auctions: Results from a pokémon field experiment," Advances in Economic Analysis and Policy, 2006.

[15] A. Andrzejak, D. Kondo, and S. Yi, "Decision model for cloud computing under SLA constraints," in IEEE/ACM International Symposium on Modelling, Analysis and Simulation of Computer and Telecommunication Systems (MASCOTS), 2010.

[16] S. Yi, D. Kondo, and A. Andrzejak, "Reducing costs of spot instances via checkpointing in the Amazon Elastic Compute Cloud," in IEEE International Conference on Cloud Computing (CLOUD), 2010.

[17] S. Yi, A. Andrzejak, and D. Kondo, "Monetary cost-aware checkpointing and migration on Amazon cloud spot instances," IEEE Transactions on Services Computing, 2011.

[18] F. M. Ortuno and U. Harder, "Stochastic calculus model for the spot price of computing power," in Annual UK Performance Engineering Workshop (UKPEW), 2010.

[19] K. Vanmechelen, W. Depoorter, and J. Broeckhove, "Combining futures and spot markets: A hybrid market approach to economic grid resource management," Journal of Grid Computing, vol. 9, pp. 81-94, 2011.

[20] J. Altmann, C. Courcoubetis, G. Stamoulis, M. Dramitinos, T. Rayna, M. Risch, and C. Bannink, "GridEcon: A market place for computing resources," in Grid Economics and Business Models, ser. Lecture Notes in Computer Science. Springer Berlin / Heidelberg, 2008, vol. 5206, pp. 185-196.

[21] M. R. Rahman, Y. Lu, and I. Gupta, "Risk aware resource allocation for clouds," University of Illinois at Urbana-Champaign, Tech. Rep., 2011. [Online]. Available: http://hdl.handle.net/2142/25754 